\title{
Evaluación del etiquetado frontal de advertencia de la Ley de Alimentos en adultos responsables de escolares de las comunas de La Serena y Coquimbo
}

\section{Evaluation of the front-of-package warning labels of the Chilean Food Law by adults responsible for schoolchildren in the districts of La Serena and Coquimbo}

\section{RESUMEN}

Una política pública creada para abordar el problema de la obesidad en Chile, es la Ley de Alimentos del año 2016 y su Reglamento que incluye etiquetado frontal de advertencia. El objetivo del estudio es evaluar el reconocimiento, juicio de valor y utilización del etiquetado frontal de advertencia descrito en la reglamentación, en personas responsables de escolares de las comunas de La Serena y Coquimbo. En estudio transversal, se aplicaron 543 encuestas personales en 22 establecimientos educacionales seleccionados por muestreo probabilístico estratificado. Se utilizó prueba de Chi-cuadrado para bondad de ajuste y tablas de contingencia, se consideró significativo un valor p<0,05. El 98,7\% reconoció los sellos de advertencia, principalmente en los envases. El 86,2\% valoró como no saludable su presencia y $68,1 \%$, como saludable su ausencia. La comparación de sellos fue realizada por el $67,5 \%$ de los encuestados, de los cuales un 97,4\% eligió los alimentos con menor cantidad de sellos. El 91,3\% señaló que disminuyó la cantidad de alimentos comprados con presencia de sellos en relación al período anterior a la vigencia de la Ley. Los sellos de advertencia son conocidos, entendidos y considerados en la selección de los alimentos.

Palabras clave: Etiquetado de alimentos; Información nutricional; Ley Chilena 20.606; Política de nutrición; Trastornos de nutrición infantil.

\footnotetext{
ABSTRACT

The Chilean Food Law of 2016 was a public policy created to address the problem of obesity in Chile and includes front-of-package warning labeling. The objective of the study is to evaluate the recognition, value judgment and use of the front-of-package warning labels described in the regulation, among caretakers of schoolchildren, in the districts of La Serena and Coquimbo, Chile. A cross-sectional study was conducted. A total of 543 personal surveys were applied in 22 educational establishments selected by stratified probability sampling. Chi-square goodness of fit test and contingency tables were used, a $p$ value $<0.05$ was considered significant. Almost all caretakers surveyed $(98.7 \%)$ recognized warning labels, mainly in packaging,
}

Natalia Alaniz-Salinas ${ }^{1}$, Mauricio Castillo-Montes ${ }^{2 *}$.

1. Secretaría Regional Ministerial de Salud Región de Coquimbo, Coquimbo, Chile. 2. Departamento de Salud Pública, Facultad de Medicina, Universidad Católica del Norte, sede Coquimbo, Coquimbo, Chile.

*Dirigir correspondencia: Mauricio Castillo-Montes. Departamento de Salud Pública, Facultad de Medicina, Universidad Católica del Norte, sede Coquimbo. Larrondo 1281, Coquimbo, Chile. Email: mcmontes@ucn.cl

Este trabajo fue recibido el 13 de febrero de 2020. Aceptado con modificaciones: 17 de junio de 2020. Aceptado para ser publicado: 23 de junio de 2020.

$82 \%$ of the participants rated the presence of the labels as unhealthy and $68.1 \%$ reported the absence of the label indicated a healthy food. The label comparison was made by $67.5 \%$ of the respondents, of which $97.4 \%$ chose foods with fewer labels. $91.3 \%$ indicated that the quantity of food purchased with warning labels decreased in relation to the period before the law was launched. Warning labels are known, understood, and considered in food selection. KEY WORDS: Child nutrition disorders; Chilean Law 20,606. Food labeling; Nutrition information; Nutrition policy.

\section{INTRODUCCIÓN}

La malnutrición por exceso, manifestada como sobrepeso y obesidad, se asocia a diversas alteraciones metabólicas, 


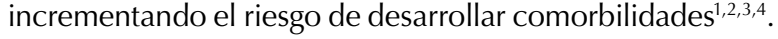
La Organización Mundial de la Salud (OMS) la declara como uno de los retos más importantes para la Salud Pública del siglo $21^{2}$. Se calcula que en América Latina y el Caribe, 3,9 millones de niños menores de 5 años tienen exceso de peso, situación que se presenta en más del 34\% de los niños bajo los 6 años atendidos en el Sistema Público de Salud de Chile ${ }^{3}$.

Los principales factores que promueven el aumento de peso y enfermedades asociadas, derivan de la exposición a un ambiente obesogénico y a las respuestas conductuales y biológicas a dicho entorno. Se destacan el consumo elevado de productos de bajo valor nutricional y alto contenido de azúcares, grasa y sodio, además de la ingesta habitual de bebidas azucaradas y la actividad física insuficiente ${ }^{1,2}$. Chile tiene el consumo más alto de bebidas azucaradas del mundo y es el segundo país de la región en ventas de alimentos ultra procesados per cápita, mientras que sólo el $14 \%$ de la población sigue tres o más recomendaciones de las guías alimentarias y sólo el $5 \%$ tendría una alimentación saludable ${ }^{3,4}$.

Desde una perspectiva alimentaria, el precio, la mercadotecnia, la disponibilidad y la asequibilidad determinan las preferencias alimentarias de las personas, las decisiones al comprar y los comportamientos alimentarios ${ }^{3}$. En este contexto, diversos países han generado acciones para combatir la pandemia de la obesidad, siendo Chile uno de ellos al promulgar la Ley 20.606 sobre Composición Nutricional de los Alimentos y su Publicidad". Ésta es conocida como "Ley de Alimentos" y entró en vigencia el 26 de junio de 2016, recogiendo las principales recomendaciones internacionales: promoviendo entornos alimentarios saludables, instalando etiquetado frontal claro y sencillo, restringiendo la publicidad de alimentos no saludables dirigida a niños y protegiendo el ambiente alimentario escolar ${ }^{6}$.

Diversos estudios ${ }^{7,8,9,10}$ indican que los sistemas de etiquetado frontal que han demostrado ser efectivos en la mejor elección de alimentos, son aquellos simples, consistentes, coloreados, que no requieren habilidades matemáticas y toman poco tiempo para ser interpretados. El etiquetado frontal de la Ley 20.606 -representado por sellos de advertencia-incluyen muchas de estas características, debido a que abarcan 4 advertencias; "Alto en" para azúcares, grasas saturadas, calorías y sodio (Figura 1), que deben estar dispuestas en la cara principal de aquellos envases de productos que contengan adición de sodio, azúcares o grasas saturadas y que superen los límites establecidos para los respectivos nutrientes y/o calorías ${ }^{5}$.

Existe variada evidencia ${ }^{3,11,12,13,14,15,16,17,18,19,20}$ que señala que las etiquetas presentadas en forma entendible, podrían tener un papel decisivo en el cambio de comportamiento del consumidor. Por otra parte, el etiquetado frontal favorece la comprensión y utilización de la información nutricional del alimento, debido a las percepciones asociadas a su existencia con esta forma de presentación ${ }^{21}$. Respecto a los sellos de advertencia que describe el Reglamento Sanitario de los Alimentos (Decreto 977/97 y sus actualizaciones) que hace aplicables las disposiciones de la Ley 20.606, se ha encontrado que son ampliamente conocidos por la población, que su presencia se asocia a características no saludables e influyen en diferentes magnitudes en la selección y adquisición de alimentos ${ }^{6,22,23,24}$.

Dada la relevancia de esta política pública, el presente estudio tiene como objetivo evaluar el componente etiquetado frontal, representado por los sellos de advertencia reglamentados, en cuanto a su reconocimiento, juicio de valor que se le otorga a la presencia y ausencia, y selección al comprar alimentos, en adultos responsables de la alimentación de niños de primero básico en las comunas de La Serena y Coquimbo. Pese a la evidencia descrita, se espera que este estudio aporte desde una visión de carácter regional, la valoración de los sellos de advertencia en la alimentación y salud de la infancia.

\section{MATERIAL Y MÉTODOS}

Se realizó un estudio descriptivo transversal, mediante un cuestionario estructurado, aplicado por entrevista a 543 personas mayores de edad y responsables del cuidado de escolares de primer año básico de establecimientos educacionales de las comunas de La Serena y Coquimbo, durante los meses de noviembre y diciembre de 2018.

\section{Muestra}

El muestreo fue aleatorio estratificado. Considerando la matrícula total de primero año de educación general básica de los establecimientos educacionales de las 2 comunas (5.185 estudiantes), se hizo una afijación proporcional de acuerdo a los tres tipos de administración educacional. En forma aleatoria simple, se seleccionaron 202 sujetos de la administración pública (9 colegios), 241 de particulares subvencionados (8 colegios) y 100 de particulares (5 colegios). La matrícula total de las 2 comunas era de $37 \%$, $45,3 \%$ y $17,7 \%$ respectivamente. Finalmente, se aplicó el cuestionario a todas las personas responsables del cuidado de los escolares que estaban disponibles y aceptaron participar.

\section{Sujetos de estudio}

Se definió como adultos responsables del cuidado, a aquellas personas encargadas de la educación y adquisición de los alimentos del escolar, sea éste familiar directo o apoderado. La invitación a participar en el estudio y la aplicación del instrumento se realizó en coordinación con los profesores jefes de los cursos, utilizando las instancias de reuniones de padres y apoderados. Se incluyó a todos los adultos hispano hablantes que aceptaron participar, encuestándose a sólo un adulto por hogar. Se categorizaron las edades en 3 grupos etarios; menores de 35, entre 35 y 50 y mayores de 50 años. Para la identificación de los sujetos, se consideró como extranjera, a aquellas personas que reportaron nacionalidad distinta a la chilena, y abuelos/ as a quienes indicaron esa relación de parentesco con el escolar al iniciar la encuesta. Quedaron fuera del estudio, 
las personas que no asistieron a la reunión de padres y apoderados y las que rechazaron participar, a quienes se les explicó que por ser un estudio voluntario, no existía ningún tipo de inconveniente ni sanción administrativa.

\section{Instrumento}

Se generó un cuestionario de 8 preguntas de selección múltiple, de una o varias respuestas, que consideró 3 dimensiones: reconocimiento, juicio de valor ante la presencia y ausencia de sellos, y su utilización en la selección de los productos alimenticios. Se realizó la validación de contenido a través de un panel de 5 expertos compuesto por; 4 profesionales nutricionistas cuyos desempeños profesionales y académicos son reconocidos en el ámbito de la Ley de Alimentos, y 1 metodólogo del área de medición por instrumentos, quienes evaluaron la encuesta mediante el índice de validez de contenido. El índice utilizado, consideró la evaluación de cada ítem por separado y de toda la encuesta en su conjunto, mediante una escala de valor 0 (no pertinente) a 3 (muy pertinente o indispensable). Posteriormente y con las respuestas, se realizaron los ajustes en el cuestionario para un segundo proceso de validación de juicio por los expertos, cuyas sugerencias de modificaciones fueron incorporadas. La etapa siguiente, consistió en una prueba piloto en un colegio particular subvencionado de enseñanza básica de la comuna de Coquimbo, que no había sido seleccionado en la muestra original. Finalmente, con los resultados de la prueba piloto, se hicieron nuevos cambios y ajustes para la encuesta definitiva (Anexo). El instrumento fue diseñado con 2 preguntas filtro $\left(N^{\circ} 1\right.$ y 6 ), que definieron la posibilidad de continuar respondiendo la encuesta o abandonarla si respondían negativamente generando 2 submuestras (Figura 2). Un total de 28 personas indicaron que no eran los encargados de comprar alimentos para los niños en el hogar, por lo tanto no fueron considerados en el análisis de las 2 últimas preguntas. Sólo la sub muestra 2 continuó respondiendo las 2 últimas preguntas de la encuesta; es decir, aquellos adultos que seleccionaron la alternativa "sí comparo la cantidad de sellos". Se capacitó

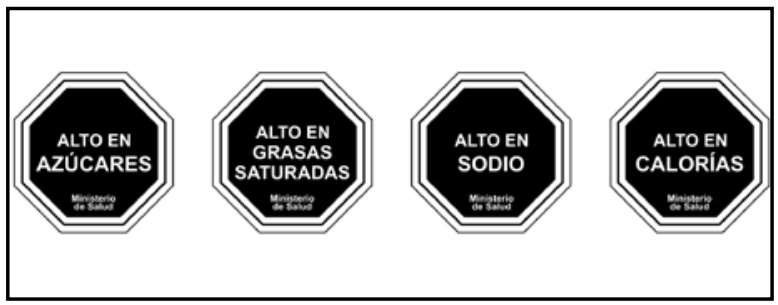

Figura 1: Sellos frontales de advertencia "altos en" de Ley 20.606 sobre Composición Nutricional de los Alimentos y su Publicidad.

Fuente: Diario Oficial de la República de Chile. 26 de junio de 2015. Modifica Decreto Supremo No 977. Reglamento Sanitario de los Alimentos. Decreto 135. y entrenó a un grupo de encuestadores para la aplicación del instrumento, quienes en forma individual, presencial y cara a cara, realizaron las preguntas en una sala destinada para este fin en un tiempo aproximado de 5 minutos.

El estudio fue aprobado por el Comité Ético Científico de la Facultad de Medicina de la Universidad Católica del Norte mediante la Resolución N No 94 del 2018, además s e solicitó la firma de consentimientos informados a los participantes y autorizaciones de directivos de los establecimientos educacionales.

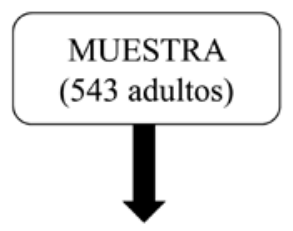

Preguntas sobre reconocimiento de sellos etiquetado frontal

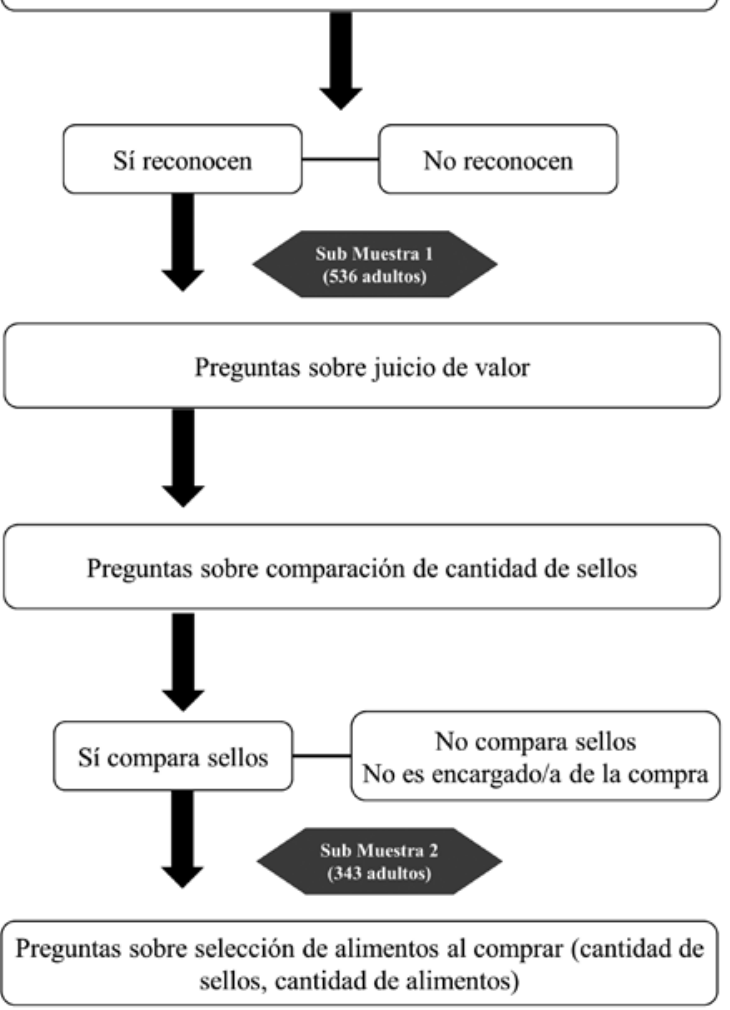

Figura 2: Diagrama de población total encuestada y sub muestras.

Anexo. Cuestionario Componente Sellos de Advertencia de Ley 20.606 


\section{ENCUESTA DE EVALUACIÓN DEL COMPONENTE "SELLOS DE ADVERTENCIA" DE LA LEY DE ALIMENTOS}

Código:

\section{Indicaciones (leidas por encuestador):}

Para contestar el siguiente cuestionario sobre los sellos de advertencia de la Ley de Alimentos, por favor seleccionar la o las alternativas, según sea el caso, que más represente su opinión y conocimiento al respecto.

Considerar que no existen respuestas buenas o malas y que se trata de un instrumento anónimo. Desde ya muchas gracias por su colaboración.

\begin{tabular}{|c|c|c|c|c|c|c|c|c|}
\hline \multicolumn{8}{|c|}{ Establecimiento Educacional: } & \\
\hline \multicolumn{9}{|c|}{ ANTECEDENTES DEL ADUITO } \\
\hline \multicolumn{2}{|l|}{ Edad: } & Género: & \multicolumn{2}{|c|}{ Femenino } & Masculino & Otro: & & \\
\hline Nacionalidad: & Chi & \multicolumn{2}{|c|}{ Extranjera } & & & & & \\
\hline \multirow{2}{*}{$\begin{array}{c}\text { Parentesco } \\
\text { con el } \\
\text { escolar: }\end{array}$} & Madre & \multicolumn{3}{|c|}{$\begin{array}{c}\text { Madrastra o pareja de } \\
\text { padre/madre }\end{array}$} & \multicolumn{2}{|c|}{ Tía/o } & Abuela/o & $\begin{array}{l}\text { Otro no } \\
\text { pariente }\end{array}$ \\
\hline & Padre & \multicolumn{3}{|c|}{$\begin{array}{c}\text { Padrastro o pareja de } \\
\text { madre/padre }\end{array}$} & \multicolumn{2}{|c|}{ Hermana/o } & $\begin{array}{c}\text { Otro } \\
\text { pariente }\end{array}$ & \\
\hline
\end{tabular}

\section{DIMENGION 1: RECONOCMMIENTO DE SELLOS}

Pregunta 1. ¿Reconoce usted los siguientes sellos? (Se muestra cartilla impresa con los 4 sellos). Elija solo 1 alternativa.

\begin{tabular}{|ll|l|}
\hline a) & Si & \\
\hline b) & No & \\
\hline
\end{tabular}
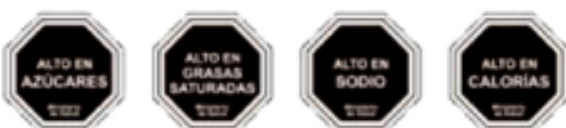

En el caso de responder "NO", finalizar el proceso de encuesta y agradecer por la participación.

Pregunta 2. ¿En qué lugar/es ha visto estos sellos? Puede elegir más de una alternativa, si corresponde.

\begin{tabular}{|ll|l|}
\hline a) & Envases de alimentos & \\
\hline b) & Reportajes de televisión, redes sociales o medios escritos & \\
\hline c) & Publicidad de alimentos & \\
\hline d) & Publicidad del Gobierno & \\
\hline e) & Actividades educativas & \\
\hline
\end{tabular}

\section{DMMENSIÓN 2: SIGNIFICANCIA NUTRICIONAL QUE SE LE OTORGA A LA PRESENCLA DE SELLOS}

Pregunta 3. ¿Qué opina usted de un alimento envasado QUE TENE sellos de advertencla? Elija solo 1 alternativa.

\begin{tabular}{|l|l|}
\hline a) Es un alimento saludable & \\
\hline b) Es un alimento no saludable & \\
\hline c) Es un alimento menos saludable en comparación a uno sin sellos & \\
\hline d) No tiene una característica nutricional definida & \\
\hline
\end{tabular}


Pregunta 4. ¿Qué opina usted de un alimento enwasado que NO TIENE sellos de advertencla? El Ija solo 1 alternativa.

\begin{tabular}{|l|l|}
\hline a) Es un alimento saludable & \\
\hline b) Es un alimento no saludable & \\
\hline c) Es un alimento más saludable en comparación a uno que presente se llos & \\
\hline d) No tiene una caracteristica nutricional definida & \\
\hline
\end{tabular}

Pregunta 5. ¿Cuál de los sellos de advertencia, considera usted, es el más importante? Elija solo 1 alternativa.

\begin{tabular}{|ll|l|}
\hline a) & Sello "ALTO EN AZÚCAR" & \\
\hline b) Sello "ALTO EN GRASASSATURADAS" & \\
\hline c) & Sello "ALTO EN SODIO" & \\
\hline d) & Sello "ALTO EN CALORIAS" & \\
\hline e) & LOS 4 sellos son importantes & \\
\hline f) & LOS 4 sellos son irrelevantes & \\
\hline
\end{tabular}

\section{DIMENSION 3: CAMBIOS EN LA CONDUCTA DE SELECCION DE PRODUCTO ALIMENTICOO EN BASEALA PRESENCIA DE SELLOS}

Pregunta 6. A la hora de comprar alimentos envasados para los nifios de su hogar, ¿Compara usted la cantidad de sellos presentes en los productos? Elija solo 1 alternativa.

\begin{tabular}{|l|l|}
\hline a) Si & \\
\hline b) No & \\
\hline c) Yo no soy e/la encargado/a de comprar alimentos para los nifnos del hogar & \\
\hline
\end{tabular}

En el caso de responder las altemativas "NO" o "Yo no soy el/a encargado/a de comprar almentos para los nilfos del hogar". finalizar el proceso de encuesta y agradecer por la participación.

Pregunta 7. Al momento de comprar alimentos envasados para los niños de su hogar ¿Considera usted la cantidad de sellos en la selección de estos alimentos? Elija solo 1 alternativa.

\begin{tabular}{|l|l|}
\hline a) Si los considero, soblo compro alimentos sin sellos & \\
\hline b) Si los considero, compro alimentos que tengan menos sellos & \\
\hline c) No los considero, compro alimentos sin importar la cantidad de sellos & \\
\hline
\end{tabular}

Pregunta 8. Al comparar las compras de alimentos envasados para los niños de su hogar que usted realizaba antes de la existencia de los sellos, ¿Ha modificado la cantidad de allmentos comprados con base en la presencia de estos sellos? Elija solo 1 alternativa.

\begin{tabular}{|l|l|l|}
\hline a) Si, ya no compro alimentos que ahora presentan sellos & \\
\hline b) Si, compro menos cantidad de los alimentos que ahora tienen sellos y antes no & \\
\hline c) No, compro la misma cantidad de los alimentos, aunque ahora tengan sellos & \\
\hline
\end{tabular}

Finalizar el proceso de encuesta y agradecer por la participación.

OBSERVACIONES DEL ENCUESTADOR: 


\section{Análisis estadístico}

Se utilizó el programa Microsoft Excel 2010 para la tabulación de los datos recolectados, los que se sintetizaron en tablas de contingencia, utilizando la prueba de Chi-cuadrado para bondad de ajuste en el análisis comparativo de las variables; se consideró significativo un valor $\mathrm{p}<0,05$. Para el análisis se usó el programa estadístico XIstat 2011.2.06.

\section{RESULTADOS}

Como se presenta en la tabla 1 , del total de 543 personas encuestadas, la mayoría fueron mujeres $(88,8 \%)$, madres $(79,2 \%)$, de nacionalidad chilena $(95,2 \%)$ y con edad promedio de $36,2 \pm 10$ años.

\section{Dimensión reconocimiento de sellos de advertencia}

El 98,7\% de los sujetos reconocieron los sellos de advertencia de la Ley de Alimentos, con menor proporción en extranjeros y en el grupo de abuelos/as. Del total de la muestra, sólo 7 personas señalaron no reconocer los sellos.

Respecto al reconocimiento de los lugares en los cuales visualizaron los sellos de advertencia, los participantes de la sub muestra 1 los identificaron de la siguiente forma y en orden decreciente: "Envases de alimentos" (98,9\%), "publicidad de los alimentos" (74,6\%), "reportajes de televisión, redes sociales o medios escritos" (72,4\%), "publicidad del gobierno" (60,1\%) y "actividades educativas" (44,0\%).

Tabla 1. Población estudiada y reconocimiento de sellos de advertencia de la Ley 20.606.

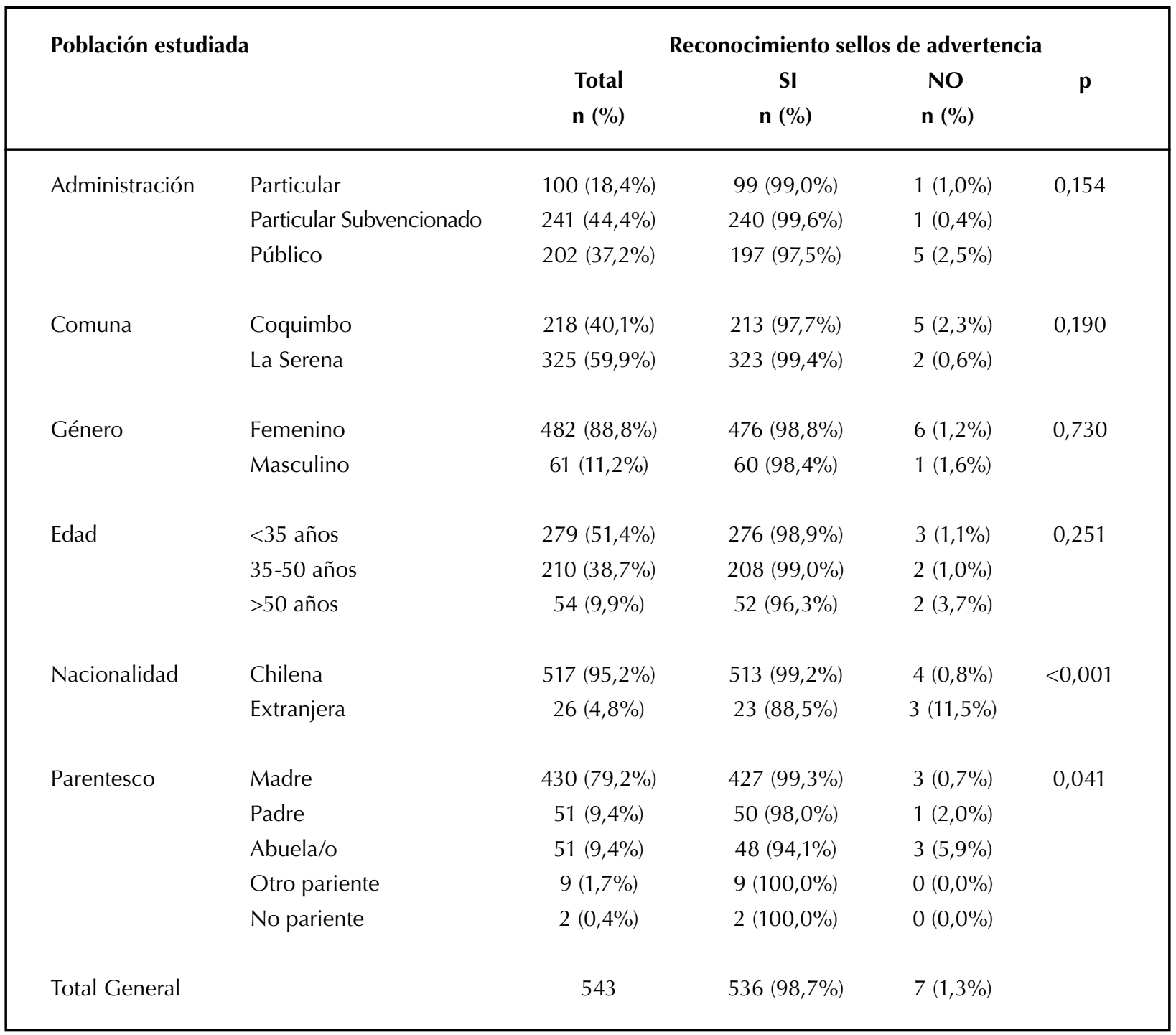

Chi-cuadrado. $\mathrm{p}<$ 0,05: existen diferencias estadísticamente significativas. Porcentajes: Población estudiada= n de cada dimensión de la variable $/ \sum \mathrm{n}$; Reconocimiento de sellos $=\mathrm{n}$ Si o No $/ \sum$ de cada dimensión de la variable. 


\section{Dimensión juicio de valor}

Al realizar las preguntas sobre opiniones en razón a que un alimento envasado tenga o no sellos de advertencia (Tabla 2), el $86,2 \%$ y el $68,1 \%$ de los entrevistados confirieron una valoración no saludable y saludable respectivamente. Se destacaron padres/madres y las personas menores de 35 años que presentaron una mayor proporción de valoración no saludable frente a la presencia de sellos. Según el tipo de administración de establecimiento educacional, los participantes de colegios particulares presentaron mayor porcentaje de valoración saludable frente a la ausencia de sellos, al igual que los menores de 35 años.
Respecto a cuál de los sellos de advertencia "alto en" era considerado como el más importante, en la tabla 3 se aprecia que en toda la muestra mayoritariamente las respuestas indicaron que todos lo eran por igual, seguido por la opción "alto en azúcares" con una proporción muy baja.

\section{Dimensión utilización de los sellos de advertencia}

La conducta sobre la toma de decisión al observar los sellos de advertencia y compra de alimentos envasados, se presenta en la tabla 4 . Se obtuvo que el $67,5 \%$ de los encuestados realizó una comparación de la cantidad de sellos. Sin embargo, las madres y personas responsables

Tabla 2. Juicio de valor sobre la presencia y ausencia de sellos de advertencia en alimentos envasados.

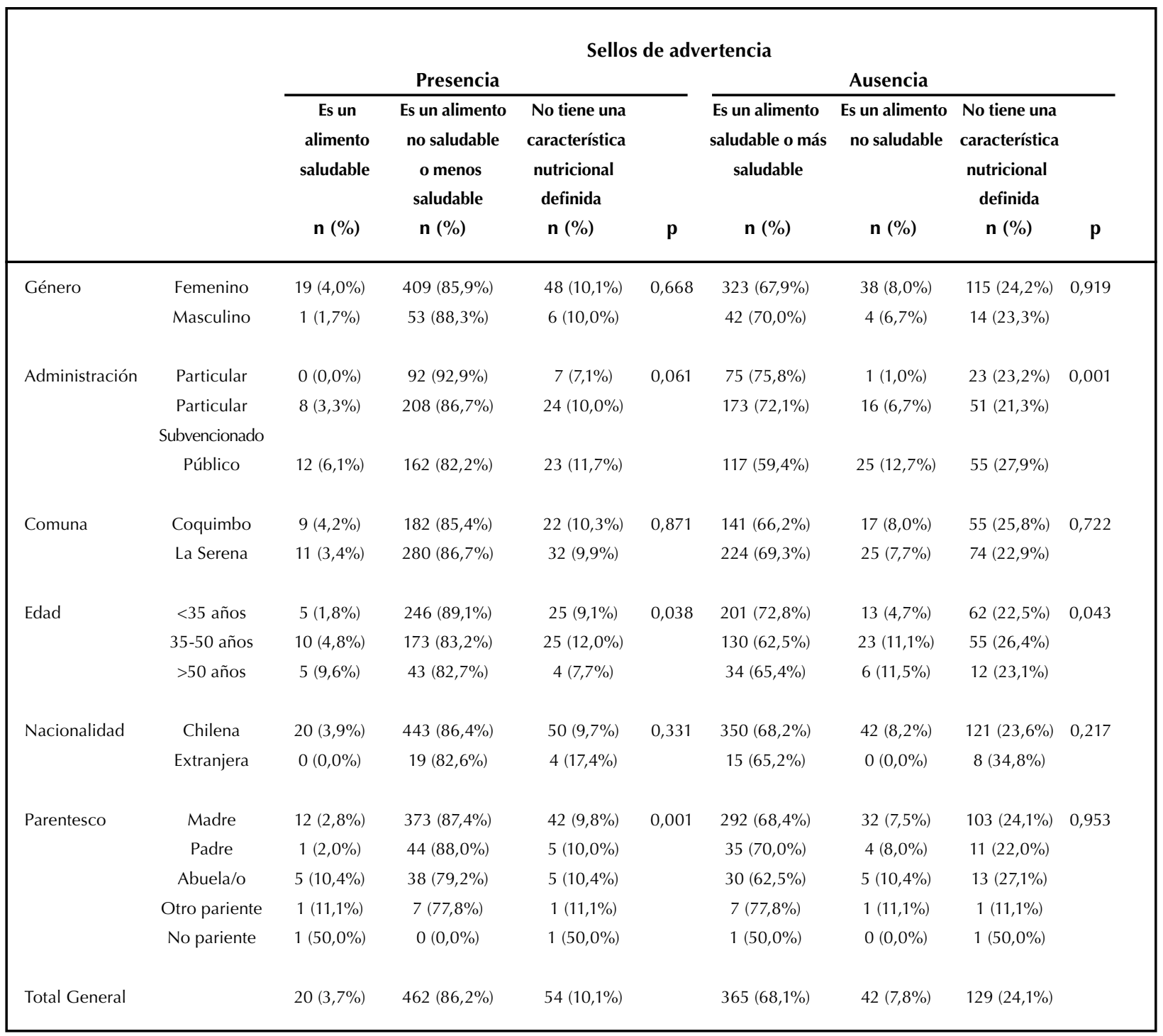


Tabla 3. Juicio de valor respecto a sello considerado como el más importante.

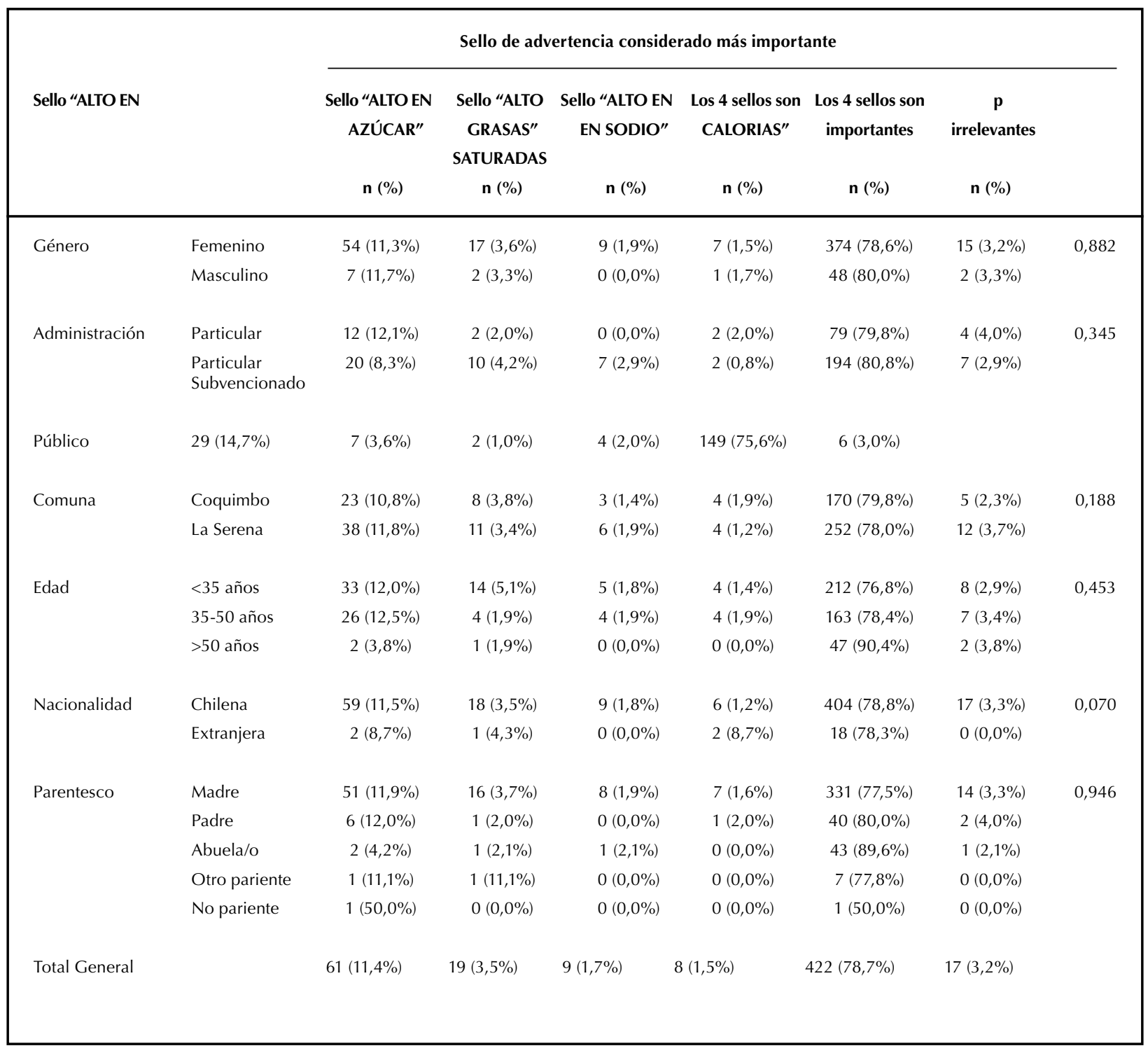

Chi-cuadrado. $\mathrm{p}<$ 0,05: existen diferencias estadísticamente significativas. Porcentajes: $\mathrm{n}$ Sello / $\sum$ de cada dimensión de la variable.

menores de 35 años fueron quienes presentaron menor frecuencia en esta práctica. El 97,1\% de la sub muestra 2 señaló que compraban alimentos con menos sellos o sin ellos, existiendo diferencias significativas en género y edad $(\mathrm{p}<0,05)$.

Así mismo, al comparar si había existido alguna modificación en la cantidad de alimentos envasados comprados en base a la presencia de sellos, versus las compras realizadas antes de la existencia de los sellos de advertencia, el 91,3\% manifestó haber reducido la cantidad de estos alimentos adquiridos.

\section{DISCUSIÓN}

El presente estudio -realizado transcurridos 2 años y medio desde la entrada en vigencia de la Ley 20.606aporta valiosos antecedentes respecto al componente sellos de advertencia. Un alto porcentaje de la población estudiada (99\%), reconoció tales sellos, situación similar a la encontrada en los estudios realizados en los años 2016 y 2017, que indicaron un nivel de reconocimiento de $92,9 \%$ y $87 \%$ respectivamente ${ }^{25,26}$. La mayor magnitud de reconocimiento se podría explicar por las múltiples acciones de difusión llevadas a cabo y el tiempo 
Tabla 4. Utilización de sellos de advertencia al comprar alimentos para los niños del hogar.

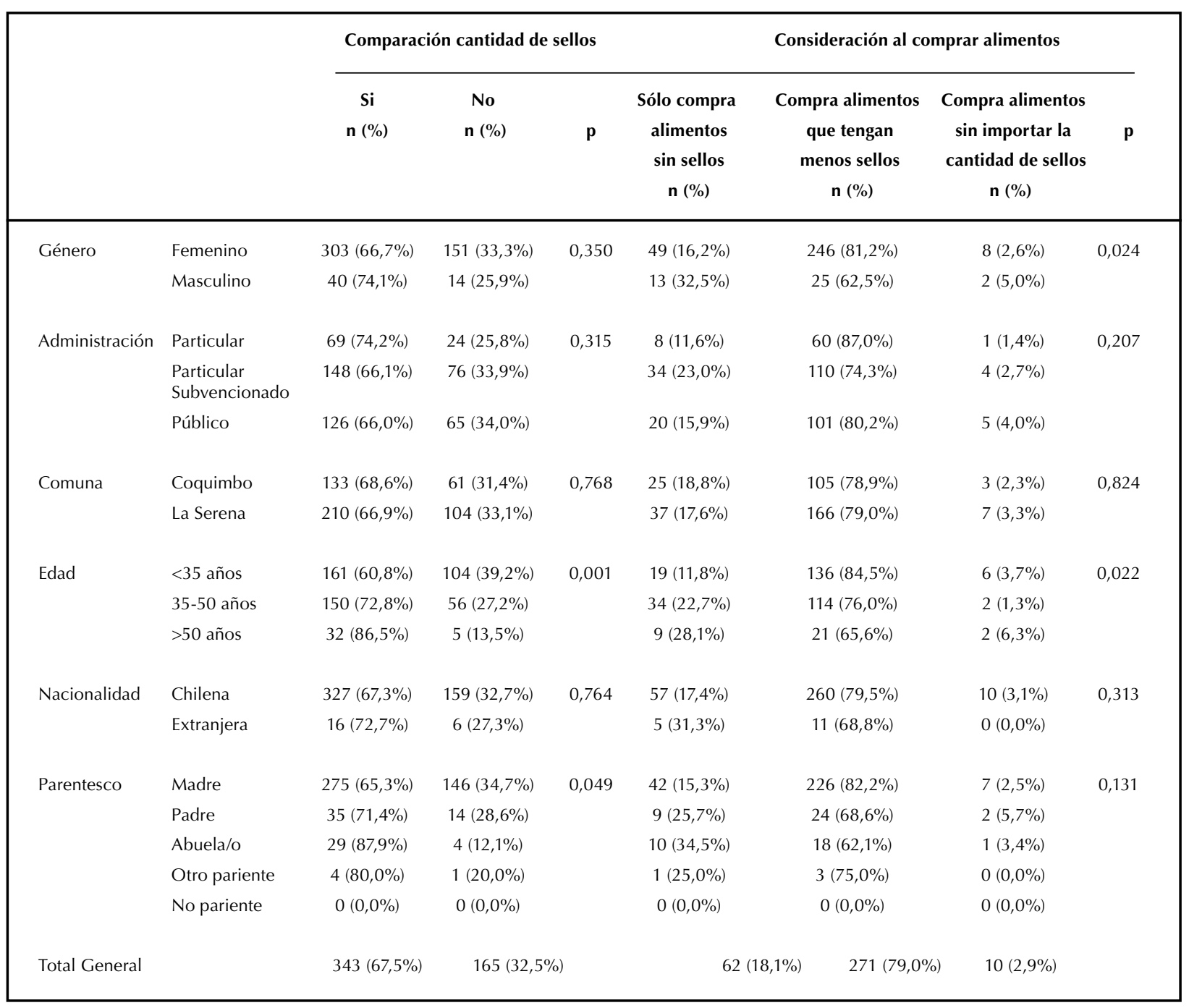

Chi-cuadrado. $\mathrm{p}<0,05$ : existen diferencias estadísticamente significativas

Porcentajes: Comparación de cantidad de sellos $=\mathrm{n} \mathrm{Si} \mathrm{o} \mathrm{No/} \sum$ de cada dimensión de la variable; Consideración de compra de alimentos= n compra alimentos $/ \sum$ de cada dimensión de la variable.

transcurrido desde la fecha de implementación de la Ley. Posiblemente relacionado a lo anterior, un hallazgo importante fue que la población extranjera reconoció los sellos en menor proporción, aspecto a considerar dada la creciente entrada de inmigrantes en Chile. De igual forma, se evidencia que el grupo de abuelas/os presentó un menor reconocimiento de sellos, antecedente similar a lo encontrado en Santiago de Chile en el año $2016^{26}$, donde los mayores de 45 años presentaron una menor proporción de reconocimiento.

Respecto a los lugares donde los sellos fueron visualizados, también existieron coincidencias con lo encontrado en el año 2016, siendo los "envases de alimentos" los principales espacios donde fueron observados, alcanzando un $99 \%{ }^{26}$. Lo anterior tiene relación con la dieta habitual de la población chilena, que se caracteriza por un amplio consumo de alimentos procesados, lo que facilita la observación de sellos.

En cuanto al juicio de valor sobre un alimento que presenta sellos de advertencia, el $86,2 \%$ de los encuestados les confirió una característica no saludable, principalmente en el grupo de padres/madres con cerca de 3 puntos porcentuales menos (91\%) que las madres de preescolares estudiadas a los 9 meses de implementada esta política ${ }^{25}$. Uno de los objetivos de la Ley 20.606 es informar a los consumidores sobre los alimentos con altas cantidades de calorías, sodio, azúcares y grasas saturadas, por su asociación con diversas enfermedades 
no transmisibles, por lo tanto este hallazgo permite establecer que la población estudiada sí entiende su significado. Sin embargo, sería de interés seguir vigilando si esta percepción tendría una tendencia a la baja como consecuencia del acostumbramiento a la alta presencia de sellos de advertencia en los alimentos que se consumen habitualmente. Diversos estudios internacionales señalan que la existencia de etiquetado frontal de advertencia, entrega información más comprensible respecto a las características nutricionales de un producto alimenticio y que la población que se encuentra expuesta a ellos, en formatos de fácil lectura como el de esta Ley, considera que los alimentos que los poseen son menos saludables y que su consumo debiera reducirse o incluso, eliminarse ${ }^{17,18,19,27,28,29}$.

En relación a la ausencia de sellos, el 68,1\% lo asoció a una característica saludable, con diferentes porcentajes entre tipos de administración de establecimiento educacional, donde los participantes de colegios privados fueron quienes seleccionaron en mayor proporción la alternativa "es un alimento más saludable en comparación a uno que presente sellos". Esta observación, es consistente con la literatura que refiere a una correlación positiva entre conocimiento nutricional y nivel socioeconómicoeducacional $^{21,30}$. Llama la atención que el $24 \%$ de los encuestados, no le confirió una característica nutricional definida a un producto sin sellos. A los 6 meses desde la implementación de esta Ley, un $71,5 \%$ de la población consideraba que estos alimentos eran saludables o de mejor calidad, mientras que el 10,5\% no tenía opinión al respecto ${ }^{26}$. Por lo tanto, en esta investigación se encontró un incremento importante de quienes no le atribuyeron una característica a la ausencia de etiquetado de advertencia. Lo anterior, debiera ser entendido considerando que en rigor la ausencia de sellos no significa necesariamente que se trate de un alimento saludable y que el propósito de la Ley es resaltar alimentos no saludables con la presencia de los sellos correspondientes. También se podría explorar algún tipo de desconfianza del consumidor hacia el etiquetado de la empresa alimentaria o porque existen otros nutrientes o componentes alimentarios que son reconocidos por la población como no saludables y que no son advertidos en esta Ley. Como ejemplo, lo planteado estaría sucediendo con las grasas trans, preservantes, colorantes y edulcorantes no calóricos, que han sido utilizados por la industria alimentaria para reemplazar en alguna proporción la presencia de azúcares en los alimentos, y de esta manera, no quedar afectos al sello "Alto en azúcares", sin embargo, parte de la población los considera como no saludables ${ }^{31}$.

Otro aspecto interesante, fue la existencia de respuestas sobre el juicio de valor que fueron inversas al mensaje que entrega la Ley de alimentos, donde el $3,7 \%$ asoció la presencia de sellos con una característica "saludable" y, sobre su ausencia, un 7,8\% le otorgó una connotación negativa o "poco saludable". Estos hallazgos pudieron deberse al desconocimiento sobre el significado de la presencia o ausencia de sellos o debido a alguna dificultad en la comprensión de la pregunta. Las respuestas a esta pregunta tuvieron frecuencias diferentes entre establecimientos educacionales, donde aquellos de la administración pública presentaron mayor proporción de respuestas inversas, seguidos por particulares subvencionados, mientras que los colegios privados obtuvieron menor número. En este sentido, la literatura manifiesta una relación directa entre conocimiento sobre alimentación y nutrición, y nivel socioeconómico, que demuestra en los niveles más desventajados, menor conocimiento de las características de los productos alimenticios, determinado por su contexto social y menor acceso a la información ${ }^{21,30,32,33}$.

En relación a la opinión sobre el sello más importante, cerca de un $78 \%$ señaló que todos son igualmente importantes, logrando sobrepasar de manera considerable a quienes habían seleccionado esta opción (39,5\%) en el año $2016^{26}$. En ambos casos el sello "alto en azúcar" logró el segundo lugar, lo que se podría explicar por la asociación que tiene la población entre este macronutriente con la obesidad y enfermedades no transmisibles como diabetes mellitus, las que son de alta prevalencia en Chile. Incluso un estudio Ilevado a cabo en escolares chilenos en el año 2016, reflejó que el grupo de nivel socioeconómico bajo consideraba que el sello "alto en azúcares" era el más importante ${ }^{34}$.

Un $67,5 \%$ de la sub muestra 1 indicó llevar a cabo una comparación de la cantidad de sellos presentes en los productos, de los cuales la gran mayoría señaló que tal acción incidió en la compra de alimentos con menos sellos o sin ellos, y en menor magnitud, eligieron menor cantidad de productos de la misma naturaleza, lo que representa resultados más favorables a los encontrados en Chile en el año $2016^{26}$. Este hallazgo debiera considerarse con cautela, pues podría tratarse de un sesgo de información producto del diseño del instrumento. Es posible que los participantes evitaron reconocer la provisión de alimentos no saludables para los menores a su cargo, a pesar de conocer sus efectos negativos en la salud, situación que representa una limitación de este estudio. El grupo de mayores de 50 años y abuelas/os, fueron los que reportaron mayor nivel de comparación, y las madres, junto a personas menores de 35 años, quienes menos lo hacían. Lo anterior se condice con un estudio realizado al poco tiempo de la entrada en vigencia esta Ley, donde quienes más comparaban correspondieron al grupo etario de 46 años o más, diferenciándose de los jóvenes entre 18 a 29 años, que comparaban menos ${ }^{25}$, lo que se podría atribuir al menor nivel de percepción de riesgo que presentarían grupos más jóvenes.

Al comparar el cambio producido en la adquisición de alimentos "altos en" en la actualidad, versus las compras de esos mismos productos antes de la entrada en vigencia de la Ley, se obtuvo una disminución 
del $91,3 \%$ en aquellos alimentos con la presencia de octágonos de advertencia. Esta cifra, llevada a la muestra total que reconoce sellos y compra alimentos para los niños del hogar, cambió al 61,6\%, lo que constituiría un importante aumento en comparación a lo encontrado en los años 2017 y 2019, donde el 26\% que respondió afirmativamente a la aseveración ¿has dejado de consumir productos porque tienen sellos $?^{25}$ y un $42,8 \%$ señaló que sí había dejado de comprar alimentos que tuvieran sellos en sus envases ${ }^{25,35}$. Esta situación se ha demostrado en estudios que han determinado la ocurrencia de cambios en la conducta de selección y, por tanto, en la venta de productos alimenticios posterior a la implementación de este tipo de política, principalmente en aquellos que presentan 2 sellos de advertencia y que serían más fáciles de sustituir en comparación a aquellos con 3 ó 118,36,37.

Una limitación de esta investigación es propia de un estudio transversal, en cuanto al posible sesgo de información anteriormente descrito. Otra limitación es que, al tener una orientación cuantitativa, reportó resultados numéricos sobre aspectos de percepción, sin explorar áreas cualitativas que podrían emanar de opiniones de los adultos responsables y que permitirían complementar, explicar e interpretar los resultados obtenidos. Por otra parte, se destaca que esta investigación fue realizada en las 2 comunas de mayor densidad poblacional de la región de Coquimbo, lo que permitió obtener valiosa información sobre valoración y prácticas del etiquetado frontal desde una perspectiva regional.

\section{CONCLUSIONES}

La población estudiada reconoce ampliamente los sellos de advertencia de la Ley 20.606, una proporción importante los asocia correctamente a características relativas a la salud y utilizan esos criterios al seleccionar los alimentos para los niños de su hogar, lo que permite establecer inicialmente, cuáles han sido los logros obtenidos desde la implementación de esta política.

Se considera la necesidad de llevar a cabo acciones focalizadas en grupo s de riesgo, correspondientes en este caso a inmigrantes, población con nivel socioeconómico más bajo y madres jóvenes, además de prestar atención a procesos de acostumbramiento que pudiesen estar afectando a la población por la amplia exposición a estos sellos. Con lo anterior, se espera que una selección informada de los alimentos, en conjunto a otras estrategias gubernamentales que deben incorporar educación y empoderamiento, se traduzcan a futuro en mejores indicadores de salud en el país.

Agradecimientos: A los directivos, personal y padres y apoderados de los establecimientos educacionales de las comunas de La Serena y Coquimbo, que accedieron a participar en este estudio. Al grupo de profesionales del panel de expertos por su importante colaboración en la validación del instrumento, a los colegas y ayudantes que llevaron a cabo el trabajo de campo y al equipo de académicos del Programa de Magíster en Salud Pública de la Universidad Católica del Norte.

Conflictos de interés: En el presente estudio no existen conflictos de interés por cuanto se trata de una Tesis de Magíster que no cuenta con financiamiento externo ni por el cual se recibirá algún tipo de compensación.

\section{BIBLIOGRAFÍA}

1. World Health Organization. Report of the Commission on ending childhood obesity. Geneva: WHO; 2016. https:// ncdalliance.org/news-events/news/ncda-and-wcrfi-welcomenew-who-report-on-ending-childhood-obesity

2. Arias-Rico J, Cortés-Cortés SM, Ramírez-Moreno E, SánchezPadilla ML, Jiménez-Sánchez RC, Saucedo-Molina $T$ de J. Childhood obesity and its relation to cardiopulmonary indicators in mexican school children. Aquichan. 2016; 16: 148-158.

3. Food and Agriculture Organization of the United Nations and the Pan American Health Organization. Panorama of Food and Nutrition Security in Latin America and the Caribbean. Santiago de Chile: FAO and PAHO; 2017.

4. Contreras-Valdez JA, Hernández-Guzmán L, Freyre MÁ. Body dissatisfaction, self-esteem, and depression in girls with obesity. Rev Mex Trastor Aliment. 2016; 7: 24-31.

5. Gobierno de Chile. Law 20,606 Nutritional Composition of Food and its Advertising. That modified the Sanitary Food Regulation, Supreme Decree 997 (1996). Official Journal of the Republic of Chile $n^{\circ} 41,193$ (june 26, 2015).

6. Food and Agriculture Organization of the United Nations and the Pan American Health Organization. Approval of a new food act in Chile: process summary. Santiago de Chile: FAO and PAHO; 2017.

7. Roberto CA, Wong D, Musicus A, Hammond D. The influence of sugar-sweetened beverage health warning labels on parents' choices. Pediatrics. 2016; 137: 1-10.

8. Scrinis G, Parker C. Front-of-pack food labeling and the politics of nutritional nudges. Law Policy. 2016; 38: 234-249.

9. Ares G, Varela F, Machin L, Antúnez L, Giménez A, Curutchet $M R$, et al. Comparative performance of three interpretative front-of-pack nutrition labelling schemes: Insights for policy making. Food Qual Prefer. 2018; 68: 215-225.

10. Shangguan S, Afshin A, Shulkin M, Ma W, Marsden D, Smith I, et al. A meta-analysis of food labeling effects on consumer diet behaviors and industry practices. Am I Prev Med. 2019; 56: 300-314.

11. Pan American Health Organization and World Health Organization. Health Situation in the Americas: Core Indicators 2016. Washington, D.C., United States of America: PHAO and WHO; 2016.

12. Ministerio de Salud. Santiago de Chile: Subsecretaría de Salud Pública; 2014 [access in march 2018]. Diagnosis of the nutritional status of children under 6 years of age, pregnant women, nurses and older adults, under control in the public health system (Diagnóstico del estado nutricional de menores de 6 años, gestantes, nodrizas y adultos mayores, bajo control en sistema público de salud); 68 pages. Available from: http:// www.minsal.cl/sites/default/.

13. Goodman S, Vanderlee L, Acton R, Mahamad S, Hammond $D$. The impact of front-of-package label design on consumer understanding of nutrient amounts. Nutrients. 2018; 10: 1-12. 
14. Graham DI, Lucas-Thompson RG, Mueller MP, Jaeb M, Harnack L. Impact of explained $v$. unexplained front-ofpackage nutrition labels on parent and child food choices: A randomized trial. Public Health Nutr. 2017; 20: 774-785.

15. Carter OBJ, Mills BW, Lloyd E, Phan T. An independent audit of the Australian food industry's voluntary front-of-pack nutrition labelling scheme for energy-dense nutrition-poor foods. Eur J Clin Nutr. 2013; 67: 31-35.

16. Ma J, Zhu Z, Chen X, Guo Y, Zhang $H$, Zhang $Y$, et al. A cross-sectional survey of nutrition labelling use and its associated factors on parents of school students in Shanghai, China. Public Health Nutr. 2018; 21: 1418-1425.

17. Talati Z, Pettigrew S, Kelly B, Ball K, Neal B, Dixon H, et al. Can front-of-pack labels influence portion size judgements for unhealthy foods? Public Health Nutr. 2018; 21: 27762781.

18. Khandpur N, Sato P, Mais LA, Bortoletto AP, Spinillo CG, Garcia $M T$, et al. Are front-of-package warning labels more effective at communicating nutrition information than traffic-light labels? A randomized controlled experiment in a Brazilian sample. Nutrients. 2018; 10: 1-15.

19. Arrúa A, Machín L, Curutchet MR, Martínez J, Antúnez $L$, Alcaire $F$, et al. Warnings as a directive front-of-pack nutrition labelling scheme: Comparison with the Guideline Daily Amount and traffic-light systems. Public Health Nutr. 2017; 20: 2308-2317.

20. United Nations Children's Fund, UNICEF. Review of current labelling regulations and practices for food and beverage targeting children and adolescents in Latin America countries (Mexico, Chile, Costa Rica and Argentina) and recommendations for facilitating consumer information. Panamá: UNICEF; 2016.

21. Anderson CL, O'Connor EL. The effect of the health star rating on consumer decision-making. Food Qual Prefer. 2019; 73: 215-225.

22. Ávalos ML, Reynoso L, Colunga C, Oropeza R, González MÁ. Relation of the body mass index, physical and sedentary activities in students. Rev Elec Psic Izt. 2014; 17: $978-996$

23. Pan American Health Organization and World Health Organization. Plan of action for the prevention of obesity in children and adolescents. Washington DC: PHAO and WHO; 2014.

24. Correa $T$, Fierro $C$, Reyes $M$, Carpentier FRD, Taillie $L S$, Corvalan C. Responses to the Chilean law of food labeling and advertising: exploring knowledge, perceptions and behaviors of mothers of young children. Int J Behav Nutr Phys Act. 2019; 16: 1-10.

25. Ministerio de Salud de Chile. Evaluation report on the implementation of the nutritional food composition law and its advertising. Santiago: Subsecretaría de Salud Pública, División de Políticas Públicas Saludables y Promoción,
Departamento de Nutrición y Alimentos; 2017.

26. Valdebenito $M$, Labrín J, León V, Fierro S. Results report: Description of consumer perceptions and attitudes regarding state measures in the framework of the implementation of Decree 13/15. Santiago de Chile: DEMOSCOPICA and Instituto de la Comunicación e Imagen ICEI; 2017.

27. Ducrot P, Méjean C, Julia C, Kesse-Guyot E, Touvier M, Fezeu $L$, et al. Effectiveness of front-of-pack nutrition labels in french adults: Results from the Nutrinet-Santé cohort study. PLoS One. 2015; 10: 1-15.

28. Talati Z, Pettigrew S, Dixon H, Neal B, Ball K, Hughes C. Do health claims and front-of-pack labels lead to a positivity bias in unhealthy foods? Nutrients. 2016; 8: $1-18$.

29. Rosenblatt $D$, Summerell $P, N g A$, Dixon H, Murawski $C$, Wakefield $M$, et al. Food product health warnings promote dietary self-control through reductions in neural signals indexing food cue reactivity. Neuroimage Clin. 2018; 18: 702-712.

30. Grunert KG, Wills JM, Fernández-Celemín L. Nutrition knowledge, and use and understanding of nutrition information on food labels among consumers in the UK. Appetite. 2010; 55: 177-189.

31. Quitral V, Arteaga, J, Rivera, M, Galleguillos, J, Valdes I. Comparison of sugar and non-caloric sweetener content in beverages before and after implementing Chilean law 20.606. Rev Chil Nutr. 2019; 46: 245-253.

32. Miller C, Wakefield M, Braunack-Mayer A, Roder D, O’Dea KO, Ettridge K, et al. Who drinks sugar sweetened beverages and juice? An Australian population study of behaviour, awareness and attitudes. BMC Obes. 2019; 6: 1-12.

33. Ducrot P, Méjean C, Julia C, Kesse-Guyot E, Touvier M, Fezeu $L K$, et al. Objective understanding of front-of-package nutrition labels among nutritionally at-risk individuals. Nutrients. 2015; 7: 7106-7125.

34. Olivares-Cortés S, Araneda-Flores J, Morales-Illanes G, Leyton-Dinamarca B, Bustos-Zapata N, Hernández-Moreno $M A$, et al. Attitudes of Chilean students from different socioeconomic levels at the beginning of the implementation of the law governing the sale and advertising of foods high in critical nutrients. Nutr Hosp. 2017; 34: 431-438.

35. Meléndez-Illanes L, Olivares S, Sáez-Carrillo K, Zapata D, Muñoz S, Granfeldt G. Actitudes de madres de preescolares ante la implementación de la ley de etiquetado nutricional en Chile. Arch Latinoam Nutr. 2019; 69: 165-173.

36. Muto T. The structure of a self-gravitating protoplanetary disk and its implications for direct imaging observations. Astrophys J. 2011; 739: 1-8.

37. Scapini $V$, Vergara $C$. The impact of the new food labeling law on the sale of products in Chile. Perfiles Económicos. 2017; 3: 7-33 